\title{
Übersetzung als Problemlösung
}

\section{Translation as Problem Solving}

Gabriela Rykalová

\begin{abstract}
The paper raises the question of which problems must be dealt with when translating phraseologisms, ad-hoc words, rare expressions, and word play. Related to the search for the best solutions when selecting a suitable expression is the problem of ,loss'. The translator is often in a dilemma about whether or not some aspects of the meaning should be omitted. In this respect translation is viewed as problem solving.
\end{abstract}

\section{Keywords}

translation, problem solving, ad-hoc-words, rare expressions, word play, allusion 


\section{1. Übersetzung als kreativer Prozess}

Das Übersetzen ist ein Prozess, bei dem ein Text als Produkt einer sprachlichen Kommunikation aus einer Ausgangssprache in eine Zielsprache übertragen wird. „Die Handlung, die der Übersetzer dabei übernimmt, besteht darin, stellvertretend für den Verfasser des ausgangssprachlichen Textes (= Ausgangstext) dessen Mitteilung an den Adressaten der Mitteilung in einem zielsprachigen Text (= Zieltext) neu zu formulieren." (KAUTZ 2002: 48f.) Dabei wählt der Übersetzer unter verschiedenen Übersetzungsstrategien, je nach Textsorte und Zweck der Übersetzung. Nach LEVÝ (1983), dem Stammvater der tschechischen Translatologie, erfolgt jede Übersetzung in drei Schritten, die einen entscheidenden Einfluss auf die Qualität der Übersetzung haben: 1) Textverständnis, 2) Textinterpretation und 3) eigene Übersetzung. Gerade in der dritten Phase des Übersetzungsprozesses muss der Übersetzer die meisten Entscheidungen treffen. In diesem Sinne ist das Übersetzen immer ein kreativer Prozess, da sich der Übersetzer auf einer ständigen Suche nach einer passenden Formulierung befindet. „Durch die Auffassung von Kreativität als Problemlösungsverfahren ist die Einsicht gegeben, dass Kreativität beim Übersetzen bereits mit dem Bewusstsein beginnt, dass ein Problem vorliegt." (CERCEL 2013: 271)

\section{2. Übersetzung als Entscheidungsprozess}

Die textlinguistische Theorie der Übersetzung bezieht sich auf die Erkenntnis, dass „Texte nicht zum Sprachsystem (langue) gehören, sondern Phänomene der Sprachverwendung (parole) in je konkreten kommunikativen Situationen sind“. (SIEVER 2015: 66) Nach dem textlinguistischen Ansatz und im Einklang mit der Sprechakttheorie steht „eine auf dem kommunikativen Effekt des gesamten Textes basierende Äquivalenz“ (vgl. ebd.) im Vordergrund des Übersetzungsprozesses.

Ein anschauliches Beispiel bilden Routineformeln. Sie haben nach BURGER (2015: 48) kaum lexikalische oder propositionale Bedeutung, sondern spielen eine konkrete Rolle in bestimmten pragmatischen Situationen. Die Verwendung der Routineformeln in bestimmten Kommunikationssituationen ist durch Konventionen, Wertsysteme und Normen vorgegeben und/oder in bestimmten Situationen als solche akzeptiert worden. Dank Konventionen und Erwartungen der Kommunikationspartner helfen sie die Handlungsakte zu vollziehen. Routineformeln sind kulturspezifisch. Die Aufgabe des Übersetzers besteht darin, die Routineformeln der Ausgangssprache durch die in der Zielsprache konventionalisierten und in bestimmten Situationen erwarteten Wendungen zu ersetzen.

Im Zusammenhang mit dem Bewusstsein von den oben genannten drei Ebenen bei der Übersetzung formuliert Kautz folgende Definition einer Übersetzung: „Übersetzen ist eine komplexe, funktional bestimmte, planmäßige, sowohl rekreative wie auch kreative Tätigkeit im Rahmen der transkulturellen sprachlichen Kommunikation zwischen verschiedensprachlichen Partnern." (KAUTZ 2002: 57) 


\section{3. Übersetzung als Problemlösung}

Auch wenn der Übersetzer beide Sprachen ausgezeichnet beherrscht, d.h. ein guter Kenner sowohl der Ausgangssprache als auch der Zielsprache ist, kann es vorkommen, dass die Übersetzung nicht problemlos durchgeführt werden muss. „Übersetzungsprobleme treten immer dann auf, wenn wir Einheiten des Ausgangstextes nicht quasi automatisch durch 1:1-Umkodierung in der Zielsprache wiedergeben können [...].“ (KAUTZ 2002: 119) Dabei sind Probleme auf drei Ebenen gemeint:

1. Probleme auf der linguistischen Ebene

2. Probleme auf der strukturellen Ebene

3. Probleme auf der kulturellen Ebene

An dieser Stelle muss betont werden, dass die Wahl der Übersetzungsmethode von zahlreichen Faktoren abhängig ist, wobei die Textsorte des Ausgangstextes eine wichtige entscheidungssteuernde Wirkung hat.

Im Folgenden werden die häufigen Schwierigkeiten bei der Übersetzung von belletristischen Texten fokussiert, und zwar im Hinblick auf die Verwendung von Phraseologismen und anderen idiomatischen Wortverbindungen, Augenblicksbildungen, Wort- und Sprachspielen und Anspielungen. Das Untersuchungskorpus, das gezielt für diese Untersuchung zusammengestellt wurde, bilden drei Bücher deutschsprachiger AutorInnen: Ich hatte mich jünger in Erinnerung: Lesebotox für die Frau ab 40 (2016) von Monika Bittl und Silke Neumayer [B/N], Das Pubertier (2014) von Jan Weiler [W] und Mein kleines Buch. Märchen, Geschichten, Zungenbrecher, Wortspiele, Gedichte (2015) von Franka Frieß. Zu den wichtigsten Kriterien für die Wahl dieser Titel gehörten das Erscheinungsjahr und der spielerische Umgang mit der Sprache. Thematisch glossieren die ersten zwei Bücher witzig aktuelle gesellschaftliche Themen, wobei sich die AutorInnen der heutigen Sprache bedienen und auch originelle, emotional aufgeladene Ausdrücke verwenden. Das dritte Buch präsentiert eine ganze Reihe von literarischen Textsorten, die auf dem Sprachspiel aufgebaut sind und interessante Beispiele für unsere Untersuchung liefern.

\subsection{Phraseologismen und idiomatische Wortverbindungen}

- Emilia gab mir auf Anordnung ihres Vaters missgelaunt ihre schlaffe Pfote und meckerte kurz über die stinkenden Blumenleichen, die wir ihr [...] mitgebracht hatten. [W:8]

- „Sieht aus wie eine Klobürste aus Eichhörnchenfell." [W:45]

- Wir sind alle raus. Aufs Abstellgleis geschoben, Generationenvertrag gekündigt. [W:45f.]

- als die Fünfzig noch Lichtjahre entfernt waren [B/N:121]

Als „Phraseologismen' bezeichnen wir feste Wortverbindungen, die sich von anderen Wortverbindungen durch die Merkmale Stabilität, Polylexikalität und Idiomatizität unterscheiden. Gemeint sind Sprichwörter (Lügen haben kurze Beine.), Redewendungen (jmdm. auf den Keks gehen), Kollokationen (eine Frage stellen, den Tisch decken), komparative 
Phraseologismen (sicher wie das Amen in der Kirche) und z.B. Routineformeln (Moin, moin). Sie „tragen zumeist in ganz besonderem Maße zur stilistischen Wirkung eines Textes bei. [...] Aufgrund ihrer spezifischen Merkmale, vor allem ihrer Bildlichkeit, aber auch der Mehrgliedrigkeit und Stabilität erweisen sich Phraseologismen nicht selten als Übersetzungsproblem.“ (FIEDLER 1999: 61) Die Übersetzbarkeit von phraseologischen Wortverbindungen aller Art hängt von mehreren Faktoren ab. In erster Linie ist es der Grad der Idiomatizität und die Kontextabhängigkeit. Von Bedeutung sind auch die kulturellen Unterschiede und die damit verbundenen Assoziationen. Probleme bei der Übersetzung treten dann auf,

- wenn die zwischensprachlichen Unterschiede zu groß sind,

- wenn sich die Phraseologismen als kulturell motiviert erweisen,

- wenn mit dem Denotat kulturhistorische Assoziationen sowie eine national-kulturelle Spezifik des sprachlichen Zeichens verbunden sind,

- wenn sich das Denotat bei Idiomen als unikal erweist (vgl. (DOBROVOL'SKIJ 1999: 48).

In unserem Untersuchungskorpus sind neben Redewendungen (jmdn. aufs Abstellgleis schieben, Lichtjahre entfernt sein) auch zahlreiche Kollokationen zu finden (den Vertrag kündigen, schlaffe Pfote). Es ist zu erwarten, dass diese präferierten Wortverbindungen in die Zielsprache ebenfalls mit präferierten Wortgruppen übersetzt werden. In Texten mit unterhaltender Funktion spielt außerdem ein bestimmter Grad an Originalität eine spezifische Rolle (wie eine Klobürste aus Eichhörnchenfell).

\subsection{Ad-hoc-Bildungen / Augenblicksbildungen}

- Und mein winzig kleiner Coolheitskredit war quasi nolens volens wieder weg. [W:23]

- Lesebotox für die Frau ab 40 [B/N],

- Emilia gab mir auf Anordnung ihres Vaters missgelaunt ihre schlaffe Pfote und meckerte kurz über die stinkenden Blumenleichen, die wir ihr [...] mitgebracht hatten. [W:8]

- Sich entspannen und wieder anfangen zu stricken. Alters-Cocooning. [B/N:12]

- Meine Was-ich-im-Leben-alles-so-noch-machen-will-Liste ist endlos lang. [B/N:10]

Als Ad-hoc-Bildungen (auch Okkasionalismen) bezeichnen wir „spontane, meist stark kontextgebundene Wortneubildungen zur Bezeichnung von neuen oder bisher nicht benannten Sachverhalten bzw. zum Ausdruck der spezifischen Einschätzung eines Referenten durch den Sprecher. Augenblicksbildungen entstehen durch kreative Anwendung von Wortbildungsregeln auf Einheiten des Lexikons.“ (BUSSMANN 2002: 105). Diese Definition sollte u.E. um das Merkmal der kreativen Anwendung von ungewöhnlichen Wortkombinationen und origineller kontextueller Einbettung erweitert werden:

- Eine verpickelte Silvesterrakete flog grußlos durch den Raum. [W:7]

- „Sieht aus wie eine Klobürste aus Eichhörnchenfell." [W:45]

- Bin ich das? Und wenn ja, warum im Knitterlook? [B/N:7] 
- Ich nenne uns einfach "Generation al dente“ - denn nicht nur unsere Nudeln sind bissfest, sondern auch wir selbst immer noch knackig. [B/N:20]

Aus den Beispielen ist ersichtlich, dass sich die Augenblicksbildungen in zwei Gruppen einteilen lassen:

1) Originelle Wortverbindungen

wie eine Klobürste aus Eichhörnchenfell,

eine verpickelte Silvesterrakete,

Generation al dente,

[ich] im Knitterlook

Bei den oben erwähnten Wortverbindungen handelt es sich um metaphorische Benennungen. Dank dieser ungewöhnlichen und überraschenden Wortkombinationen von ganz gewöhnlichen Wörtern (Ad-hoc-Wortkombinationen) entstehen bestimmte, eine Komik erzeugende Assoziationen. Dank der Tatsache, dass Metaphern auf Bildern beruhen, ermöglichen sie dem Übersetzer, ähnliche Bilder auch in der Zielsprache zu schaffen.

2) Originelle Wortbildungen

Eine viel größere Herausforderung bilden okkasionell gebildete Komposita aller Art $[\mathrm{B} / \mathrm{N}]$ :

Lesebotox / Coolheitskredit / Blumenleichen / Ärztetourismus / Kuschelparty / Schnäppchenroute / Hungermodels / Couchsurfen / endpeinlich

Eine Besonderheit der deutschen Wortbildung ist die im Deutschen zunehmende Präferenz von Bindestrichkomposita, die oft aus unterschiedlich langen Zusammenrückungen bestehen $[\mathrm{B} / \mathrm{N}]$ :

\author{
Aging-Voodoo / Ü-50-Frau / Ü-50-Party / Alters-Cocooning \\ Männerkontakt-Kneipenkosten-Quote \\ Männerkontakt-Onlinedating-Portalkosten-Quote \\ Teilzeit-Familien-Kinder-Falle \\ Eine Was-ich-im-Leben-alles-so-noch-machen-will-Liste
}

Da es sich im Falle von Augenblicksbildungen um einmalige und originelle Wortschöpfungen handelt, liegt auf der Hand, dass es bei der Übersetzung höchstwahrscheinlich um in der Zielsprache nicht existierende Wörter und Wortkombinationen geht. Einige Sprachen sind sprachtypologisch so unterschiedlich, dass der Übersetzer auf das Problem der unterschiedlichen Sprachsysteme der Ausgangs- und der Zielsprache stoßen kann (wie es im Falle des Deutschen und des Tschechischen ist). Im Deutschen ist die häufigste Wortbildungsart die Komposition, was aber nicht auf die stark flektierende 
tschechische Sprache zutrifft. Deswegen werden deutsche Komposita ins Tschechische am häufigsten als Mehrwortbenennungen übersetzt (vgl. auch KOTŮLKOVÁ 2009). Wenn man sich die oben angeführten Belege anschaut, ist nicht zu bezweifeln, dass der Übersetzer ein Problem zu lösen hat.

\subsection{Wort- und Sprachspiele}

Mit der Entscheidung zwischen unterschiedlichen Übersetzungsalternativen ist auch die Übersetzung von polysemen Wörtern sowie Homophonen und Homographen verbunden:

Jsem z východu. - I'm from exit.

Tvé oči zář́ - You eyes September.

Nebud' labut! - Don't wake up a swan!

Odpočívej v pokoji. - Relax in livingroom.

Quelle: [URL1]

Diese nicht aus unserem Korpus stammenden Beispiele sollen veranschaulichen, dass die Homonyme überhaupt eine Quelle von zahlreichen Wort- und Sprachspielen sind (z.B. in der Werbung), dass sie aber zugleich eine wahre Herausforderung für den Übersetzer darstellen und nicht selten auch eine Quelle von Übersetzungsfehlern bilden können. Viele Sprachspiele entstehen dank einer Polysemie. Dadurch, dass ein Ausdruck doppeldeutig ist, ermöglicht er mehrere Interpretationen, die komisch wirken können. Die Übersetzung von Wortspielen ist aus dem Grund schwierig, dass „sie zwei (oder mehr) Ausdrücke in Opposition zueinander setzen, die unterschiedliche Bedeutungen, aber die gleiche oder eine ähnliche Form haben“ (SNELL-HORNBY/HÖNIG/KUSSMAUL/SCHMITT 2006: 285):

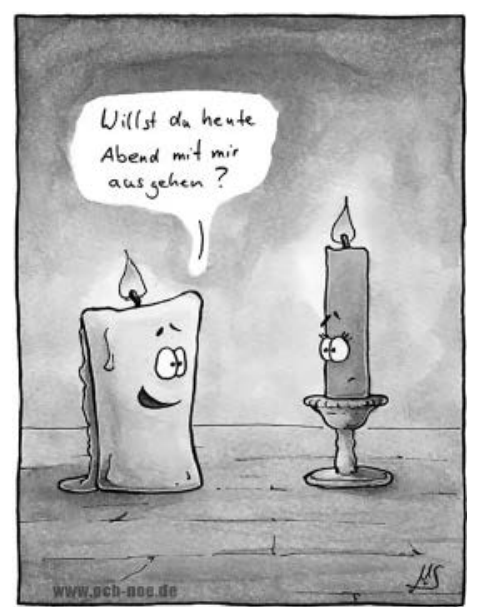

Quelle: [URL2] 
„Einen Typ für sich, obwohl mit dem Abecedarius durch das Akronym-Prinzip verwandt, stellt der ,Pangrammatismus“, auch ,Tautogramm“ oder ,Paramoion“ genannt, dar, d.h. ein Buchstabenspiel, das darin besteht, in einem Textteil oder einem ganzen Text ausschließlich (oder wenigstens möglichst viele aufeinanderfolgende) Wörter mit demselben Initialbuchstaben zu verwenden, ein Verfahren, das aus der Antike stammt [...] (ULRICH 1997: 101).

\section{Meine melodiöse Mama Moni}

Meine melodiöse Mama, Moni mit Namen, mag morgens Müsli mit Marmelade und Marmorkuchen. Mmh! Mein musikalischer Papa, Moritz mit Namen, mag morgens Mehlklößchen mit Magerquark und Moselwein. Mmh? Meine mutige Oma, Maria mit Namen, mag morgens Mus mit Mirabellen und Maronenpüree. Mmh? Meine Schwester, Miriam mit Namen, mag morgens Mandelbrot mit Mettwurst und Marzipan. Mmh? Meine Wenigkeit, Marlenchen mit Namen, mag morgens Melone mit Mandarinen und Mohnschnecken. Mmh! Mein Mops, Mimi mit Namen, mag morgens alles zusammen. Brr!

Quelle: (FRIESS 2015: 37)

\section{Milada}

Milada má malou mzdu. Má matku mlékařku malé mistni mlékárny. Musi mnoho makat. Matka má mimo jiné moc mrňavoučký močový měchýř, mononukleózu, mor. Musí maskovat mnoho měsícu maloby. Myers miluje mícové mače. Myerse muči migréna malého mozku. Milada maže Myerse měsíckovou mastí. Migréna mizi. Myers má malou, miniaturni modřinu. Macatá Milada mile mrkla. Myers má milostné mrazení. „Má Milado, miluji mladé maso. “ Milada má mravné myšlenky. „Má matka marodí, marně mne miluješ. Milado má mysl myslí mimo mne. Mám movitý majetek, miliony, mercedesy, miláčk, matka může mít misto marodění mé milióny, márnice matku mine, " mluvil měkce. Mám mámenı? Mysli Milada. Miluje mne?

Quelle: [URL1]

Es hängt von der Art des Sprachspiels und den Möglichkeiten der Zielsprache ab, ob ein Teil eines Sprachspiels oder sogar das ganze Sprachspiel geopfert werden muss, oder ob es dem Übersetzer gelingt, den Text mit den gleichen oder ähnlichen Effekten in die Zielsprache zu übertragen. (vgl. RYKALOVÁ 2017: 234)

\subsection{Anspielungen}

„Das Charakteristikum von Anspielungen [...] ist, dass sie sich immer auf etwas textuell schon Vorhandenes beziehen.“ (WILSS 1989: 3) Die Anspielungen besitzen einen hohen Originalitätsgrad und sind assoziationszentriert. Unter der Voraussetzung, dass sie vom Textrezipienten erkannt werden, d.h. dass sein Vorwissen aktiviert wird, können sie einen Überraschungseffekt auslösen. Im engeren Sinne können als Anspielungen auch einige Wortspiele und okkasionelle Wortbildungen verstanden werden, wie z.B: Inline-Bekanntschaft [B/N: 123], als eine Anspielung auf eine Online-Bekanntschaft oder 
Was-ich-im-Leben-alles-so-noch-machen-will-Liste [B/N: 10], als eine Anspielung auf die z.Z. auch in der deutschen Sprache schon ziemlich gebräuchliche To-do-Liste.

Das Problem bei einer Übersetzung liegt in der Tatsache, dass viele Anspielungen auf kulturellen und/oder historischen Traditionen und Hintergründen beruhen. Die Grenzen zwischen Kulturen werden auf der einen Seite als unscharf und kaum bemerkbar, auf der anderen Seite als fest und unüberbrückbar empfunden. Übersetzungen sind Übertragungen mittels einer Sprache. Übertragungen zwischen zwei Sprachen und Kulturen. Der Übersetzer tritt „[...] nicht nur als Sprach-, sondern auch als Kulturvermittler hervor, der durch die von ihm getroffenen Entscheidungen seine eigene Rolle bei der Übertragung von Literatur, sowie die Rolle von übersetzter Literatur im Ausland definiert“ (LETAWE 2014: 139). Wie bereits gesagt, geht es bei Anspielungen um die Übertragung von Assoziationen, die im Text nicht direkt benannt werden. Oft wird über die Beziehung zwischen dem Gesagten, dem Nicht-Gesagten und dem Gemeinten nachgedacht: „Das Gesagte und das Gemeinte sind aber in einem Text keineswegs hermetisch voneinander getrennt. Vielmehr stehen sie in einem besonderen Zusammenhang, in dem das eine das andere bestimmt und umgekehrt.“ (CERCEL 2013: 199).

\section{Fazit}

Wie zu sehen ist, trifft für Übersetzungen von Phraseologismen, Wortspielen, Anspielungen und okkasionellen Neubildungen die von KUSSMAUL (2000: 17) geprägte Bezeichnung der Übersetzung als ,kreative Leistung، in einem besonderen Maße zu. Nur selten, soweit es die Sprachsysteme beider Sprachen und die Sprachnormen erlauben, können Sprachelemente der Ausgangssprache eins-zu-eins durch Sprachelemente der Zielsprache ersetzt werden (vgl. KUSSMAUL 2000: 21). Viel häufiger müssen die Übersetzer die Inhalte der Zielsprache anpassen und dabei kleinere oder größere Probleme lösen. Aus diesem Grund stehen die Begriffe ,Problemlösung ‘ und ,Kreativität‘ in einem engen Zusammenhang.

Die Phraseologismen, Wortspiele, Anspielungen und okkasionellen Neubildungen können im Text unterschiedliche Funktionen haben. Sie dienen z.B. einer Ausdrucksverstärkung, Informationskondensierung, dem Ausgleich von Bezeichnungslücken, aber vor allem schaffen sie stilistische Effekte (vgl. auch BUSSMANN 2002: 105). Auch wenn unter den oben erwähnten Umständen der Zieltext mit dem Ausgangstext nie identisch sein kann, da die Äquivalenz nicht auf allen Ebenen erreichbar ist, sollte sich der Übersetzer bei der Übersetzung um das Erzielen von den gleichen stilistischen Effekten unter der Berücksichtigung der Intention des Autors und der Funktion des Ausgangstextes bemühen. Obwohl die oben dargestellten Herausforderungen nur im Zusammenhang mit der Übersetzung belletristischer Texte besprochen wurden, kann gesagt werden, dass sie auf alle Texte, in denen sich der Autor um eine originelle Ausdrucksweise und einen spielerischen Umgang mit der Sprache bemüht, zutreffen. 


\section{Quellen}

[B/N]: BITTL, Monika/NEUMAYER, Silke (2016): Ich hatte mich jünger in Erinnerung: Lesebotox für die Frau ab 40. München: Knaur.

FRIESS, Franka (2015): Mein kleines Buch. Märchen, Geschichten, Zungenbrecher, Wortspiele, Gedichte. Norderstedt.

[W]: WEILER, Jan (2014): Das Pubertier. Reinbek bei Hamburg: Rowohlt Verlag.

[URL1]: https://plus.google.com/104700367274544121107/posts/94qktKUps9p

[URL2]: https://www.google.cz/search?q=willst + du+heute+abend\&source=lnms\&tbm=isch\&sa $=\mathrm{X}$ \&ved=0ahUKEwiMzom0sfnaAhUJDsAKHeFLAMwQ_AUICigB\&biw=1659\&bih=890\#imgrc $=\mathrm{C}$ uCgubE9JwA9ZM: (8.5.2018)

[URL3]: http://www.povidky-az.wz.cz/m.php (1.9.2017)

\section{Literatur}

BURGER, Harald (2015): Phraseologie. Eine Einführung am Beispiel des Deutschen. Berlin: Erich Schmidt Verlag.

BUSSMANN, Hadumod (2002): Lexikon der Sprachwissenschaft. Stuttgart: Alfred Kröner Verlag. CERCEL, Larisa (2013): Übersetzungshermeneutik. Historische und systematische Grundlegung. St. Ingbert: Röhrig Universitätsverlag.

DOBROVOL'SKIJ, Dmitrij (1999): Kulturelle Spezifik in der Phraseologie. In: SABBAN, Annette (Hg.): Phraseologie und Übersetzen. Phrasemata II. Bielefeld: Aisthesis Verlag, S. 41-58.

FIEDLER, Sabine (1999): Zum Übersetzen von Phraseologismen in der Plansprache. In: SABBAN, Annette (Hg.): Phraseologie und Übersetzen. Phrasemata II. Bielefeld: Aisthesis Verlag, S. 59-80.

KAUTZ, Ulrich (2002): Handbuch Didaktik des Übersetzens und Dolmetschens. 2. Aufl. München: Iudicium.

KOTU゚LKOVÁ, Veronika (2009): Deutsche Determinativkomposita und ihre Äquivalente im Tschechischen. Saarbrücken: Südwestdeutscher Verlag für Hochschulschriften.

KUSSMAUL, Paul (2000): Kreatives Übersetzen. Tübingen: Stauffenburg.

LETAWE, Céline (2014): Über Möglichkeiten und Grenzen des Kulturtransfers. In: PASEWALCK, Silke/NEIDLINGER, Dieter/LOOGUS, Terje (Hg.): Interkulturalität und (literarisches) Übersetzen. Tübingen: Stauffenburg Verlag Brigitte Narr, S. 129-139.

LEVÝ, Jiří (1983): Umění překladu. Praha: Odeon.

PARIANOU, Anastasia (1999): Routineformeln und ihre kulturelle Einbettung - unter besonderer Berücksichtigung des Sprachenpaares Deutsch - Griechisch. In: SABBAN, Annette (Hg.): Phraseologie und Übersetzen. Phrasemata II. Bielefeld: Aisthesis Verlag, S. 175-186.

PASEWALCK, Silke/NEIDLINGER, Dieter/LOOGUS Terje (Hg.) (2014): Interkulturalität und (literarisches) Übersetzen. Tübingen: Stauffenburg Verlag Brigitte Narr.

RYKALOVÁ Gabriela (2017): Wortspiele und idiomatische Wortverbindungen als harte Nuss für Übersetzer. In: ACC Journal 3/2017, S. 227-235.

SABBAN, Annette (Hg.) (1999): Phraseologie und Übersetzen. Phrasemata II. Bielefeld: Aisthesis Verlag. 
SIEVER, Holger (2015): Übersetzungswissenschaft. Eine Einführung. Tübingen: Narr Francke Attempto Velag.

SNELL-HORNBY, Mary/HÖNIG, Hans G./KUSSMAUL, Paul/Schmitt, PETER A. (Hg.) (2006): Handbuch Translation. Tübingen: Stauffenburg.

ULRICH, Miorita (1997): Die Sprache als Sache. Primärsprache, Metasprache, Übersetzung. Tübingen: Gunter Narr Verlag.

WILSS, Wolfram (1989): Anspielungen. Zur Manifestation von Kreativität und Routine in der Sprachverwendung. Tübingen: Max Niemeyer Verlag.

Dieser Beitrag entstand mit der Unterstützung des Projekts der Schlesischen Universität in Opava SGS/4/2018, Text Analysis and Interpretation“,Analyza a interpretace textu“.

doc. PhDr. Gabriela Rykalová, Ph.D. / gabriela.rykalova@fpf.slu.cz

Slezská univerzita v Opavě, Filozoficko-přírodovědecká fakulta,

Ústav cizích jazyků, oddělení germanistiky, Masarykova třída 37, 74601 Opava, CZ 